\title{
Serum free light chain levels and renal function at diagnosis in patients with multiple myeloma
}

\author{
Punit Yadav ${ }^{1,2}$, Paul Cockwell ${ }^{1,2}$, Mark Cook³, Jennifer Pinney ${ }^{1}$, Hannah Giles ${ }^{2}$, Yu Sandar Aung ${ }^{3}$, David Cairns ${ }^{4}$, \\ Roger G. Owen ${ }^{5}$, Faith E. Davies ${ }^{6,7}$, Graham H. Jackson ${ }^{8}$, J. Anthony Child ${ }^{4}$, Gareth J. Morgan ${ }^{6,7}$ \\ and Mark T. Drayson $2,9^{*}$
}

\begin{abstract}
Background: Renal impairment (RI) is common in multiple myeloma (MM) and is associated with poor survival. This study reports the associations between renal function and disease characteristics including serum free light chain (FLC) level at diagnosis in patients with MM.

Methods: Using data from the Medical Research Council Myeloma IX trial, a multicentre, randomized, open-label, phase III and factorial-design trial, we assessed the relationships between renal function, demographic, and disease characteristics, including serum FLC levels, in 1595 newly diagnosed MM patients. Multivariable linear regression was utilised to identify factors that were associated with renal function at diagnosis. A receiver operating characteristic curve (ROC) was used to identify the optimal threshold for serum FLC level at diagnosis to predict severe RI.

Results: $52.8 \%$ of patients had an estimated glomerular filtration rate (eGFR) $\geq 60 \mathrm{ml} / \mathrm{min} / 1.73 \mathrm{~m}^{2}$ (no Rl), $37.3 \%$ an eGFR 30-59 ml/min/1.73 $\mathrm{m}^{2}$ (mild to moderate RI), and 9.8\% an eGFR $<30 \mathrm{ml} / \mathrm{min} / 1.73 \mathrm{~m}^{2}$ (severe RI). In a multivariable analysis, factors independently and negatively associated with eGFR at diagnosis were: higher serum FLC level, female gender, and older age. Elevated serum FLC level at diagnosis, irrespective of the paraprotein type, was strongly associated with severe RI. Receiver operating characteristic curve analysis showed a serum FLC level of > $800 \mathrm{mg} / \mathrm{L}$ as the optimal cut-off associated with severe RI (area under curve $0.86,95 \%$ confidence interval 0.77-0.84).

Conclusion: There was a strong relationship between higher serum FLC levels at diagnosis and the severity of Rl that was irrespective of the paraprotein type. We report an increased risk of severe RI in patients presenting with serum FLC levels above $800 \mathrm{mg} / \mathrm{L}$ at diagnosis.
\end{abstract}

Keywords: Myeloma, Serum free light chain level, Renal impairment

\section{Background}

Renal impairment (RI), as defined by an estimated glomerular filtration rate (eGFR) of less than $60 \mathrm{ml} / \mathrm{min} / 1.73 \mathrm{~m}^{2}$, is present in up to $50 \%$ of patients with multiple myeloma $(\mathrm{MM})$ and is associated with a poor prognosis $[1,2]$. Renal impairment in MM can be multi-factorial; long-standing RI can be attributed to age-related co-morbidities such as hypertension and diabetes mellitus and is largely irreversible

\footnotetext{
* Correspondence: m.t.drayson@bham.ac.uk

${ }^{2}$ Institute of Immunology and Immunotherapy, University of Birmingham, Birmingham, UK

${ }^{9}$ Clinical Immunology Service, College of Medical and Dental Sciences, University of Birmingham, Edgbaston, Birmingham B15 2TT, UK

Full list of author information is available at the end of the article
}

$[3,4]$. Acute kidney injury (AKI) can be secondary to concurrent infections, dehydration, hypercalcaemia and use of nephrotoxic drugs and is potentially reversible with timely supportive care. The commonest cause of severe RI at diagnosis (estimated glomerular filtration rate (eGFR) $<30 \mathrm{ml} /$ $\mathrm{min} / 1.73 \mathrm{~m}^{2}$ ) is through nephrotoxicity of the secreted immunoglobulin free light chain (FLC); this can be potentially reversed by the rapid lowering of the involved FLC with effective anti-myeloma therapy [5-7].

Up to one in five patients with MM have severe RI at diagnosis, this is ten-fold the prevalence of severe RI in an age- and gender-matched population from which MM arises [8, 9]. Approximately $4 \%$ of patients have RI

(C) The Author(s). 2018 Open Access This article is distributed under the terms of the Creative Commons Attribution 4.0 International License (http://creativecommons.org/licenses/by/4.0/), which permits unrestricted use, distribution, and 
that requires dialysis treatment [10]. Patients with MM and severe RI have a risk of death that is at least two-times higher than patients with MM and normal renal function [11].

Renal biopsy series from patients with MM have been restricted to individuals with severe RI, often requiring dialysis, and up to $90 \%$ of patients in these series have myeloma cast nephropathy (MCN) [6]. Myeloma cast nephropathy occurs because in some patients the FLC secreted by the neoplastic plasma cells are both at a high level and are nephrotoxic. Renal biopsies are rarely carried out in patients who do not have severe RI. It is hence not known what proportion of the mild to moderate RI in MM at diagnosis is attributed to nephrotoxicity of the secreted FLC, to other myeloma-related causes of renal damage or to the longstanding RI that develops due to other unrelated comorbidities. Outlining the relationship between the serum FLC level and renal function is important, as it has the potential to provide a mechanistic basis for the development of RI which may in turn direct future changes in treatment in MM based on the FLC levels and renal function at presentation.

In this study, we assessed the relationship between serum FLC levels and renal function across all stages of RI, except for patients receiving or likely to require dialysis, and analysed for a threshold level in serum above which there is a greater likelihood of FLC induced nephrotoxicity in patients with MM. We utilised the Medical Research Council (MRC) Myeloma IX trial dataset and included patient demographics and disease parameters, with a focus on paraprotein types and serum FLC levels at diagnosis, and renal function as measured by eGFR.

\section{Methods}

The MRC Myeloma IX trial was a multicentre, randomized, open-label, phase-III, and factorial-design trial conducted in the United Kingdom (International Standard Randomised Controlled Trial Number 68454111). Trial protocol details have been published previously [12-15]. In brief, patients aged 18 years or older with symptomatic new MM were eligible to participate in the study. Exclusion criteria included pregnancy, asymptomatic MM, solitary bone plasmacytoma or extramedullary plasmacytoma, previous or concurrent active malignancies, and presence of severe AKI unresponsive to up to 72-h of rehydration, characterised by a serum or plasma creatinine more than $500 \mu \mathrm{mol} / \mathrm{L}$, a urine output less than $400 \mathrm{ml} /$ day, or a requirement for dialysis. A multicentre research ethics committee and local ethics committees approved the protocol and all patients gave written informed consent in accordance with the Declaration of Helsinki.

All patients recruited in the intensive and non-intensive treatment arms of the MRC Myeloma IX trial were eligible to participate in this study if blood samples had been sent for central laboratory analysis at entry to the trial. The two induction regimens: oral cyclophosphamide, thalidomide, and dexamethasone (CTD) treatment were compared with infusional cyclophosphamide, vincristine, doxorubicin, and dexamethasone (CVAD) in patients on intensive treatment arm. Patients in the non-intensive treatment arm were randomised to receive either melphalan and prednisolone versus attenuated CTD. The variables that were measured at diagnosis included: age, gender, serum creatinine $\left(\right.$ Roche $\left.^{\circ}\right)$, paraprotein type $\left(\mathrm{Sebia}^{\circ}\right)$, paraprotein level, and serum FLC level (Freelite assays from The Binding Site Ltd., Birmingham, UK). The eGFR was calculated using the Modification of Diet in Renal Disease (MDRD) formula: eGFR $\left(\mathrm{ml} / \mathrm{min} / 1.73 \mathrm{~m}^{2}\right)=186 \mathrm{x}$ (Serum creatinine / 88.4 $)^{-1.154} \times(\text { Age })^{-0.203} \times(0.742$ if female gender) $\mathrm{x}$ (1.210 if black ethnicity). Patients were divided into three categories based on their eGFR at diagnosis; these categories were based on those used in clinical practice for the classification of chronic kidney disease (CKD): no RI (eGFR $\geq 60 \mathrm{ml} / \mathrm{min} / 1.73 \mathrm{~m}^{2}$ ), mild to moderate RI (eGFR $30-59 \mathrm{ml} / \mathrm{min} / 1.73 \mathrm{~m}^{2}$ ) and severe RI (eGFR $<30 \mathrm{ml} / \mathrm{min} /$ $1.73 \mathrm{~m}^{2}$ ). Renal biopsy data was not collected as part of the MRC Myeloma IX study protocol; hence results of biopsy findings if performed for any patient recruited in the study were unavailable for analysis.

Statistical analysis was performed using SPSS $^{\circ}$ for Windows, version 21.0 (SPSS Inc., Chicago, IL) and Graph Pad Prism 5.0 (GraphPad Software Inc., San Diego, CA). Categorical variables were summarised as frequencies and percentages. Comparisons for categorical variables among different groups were made with the chi-square test and Fisher's exact test where appropriate. Continuous variables were expressed as mean and standard deviation (SD) if the data were normally distributed or median with interquartile range (IQR) for non-normally distributed data. Kruskal-Wallis test was used to compare differences in age, involved kappa FLC level, involved lambda FLC level, involved FLC level as per paraprotein type and eGFR at diagnosis by eGFR categories. Mann-Whitney $U$ test was used to compare the differences in eGFR by gender and treatment arms at diagnosis based on eGFR categories. Differences in the involved serum FLC level at diagnosis for the two FLC isotypes by eGFR categories were also compared by the Mann-Whitney $U$ test. Association between serum FLC level categories and eGFR categories was assessed by the chi-square test. The association between FLC level in serum and severe RI was evaluated by the receiver operating characteristic (ROC) curve analysis. The area under the curve (AUC) of sensitivity was plotted against 1-specificity and was reported with a 95\% confidence interval (CI). ROC curve analysis was used to determine the optimal cut-off point that maximized the 
threshold value with the highest specificity and sensitivity for severe RI. Spearman's correlation coefficient $\left(r_{s}\right)$ was calculated to evaluate the relationship between age and $\log _{10}$ transformed FLC and eGFR. A multivariable linear regression analysis was performed with factors that were independently associated with eGFR at diagnosis.

\section{Results}

\section{Patient demographics and renal function}

1966 patients with newly diagnosed symptomatic MM were recruited in the MRC Myeloma IX trial; 1112 (56.6\%) were assigned to the intensive arm and 854 (43.4\%) to the non-intensive arm of the trial (Fig. 1). Renal function from samples sent for central laboratory analysis at diagnosis were available in 1595 patients who were the focus of attention of this study. Out of these 1595 patients, 907 patients were from the intensive arm and 688 patients from the non-intensive arm. The remaining 371 patients had no samples sent for central laboratory analysis at diagnosis and were excluded from analysis in this study.

Table 1 shows the baseline patient characteristics. There was a significant association between age at diagnosis and the severity of RI $(P<0.001)$; patients with RI were older compared to patients with no RI. There was no difference in median eGFR by gender in patients presenting with mild to moderate $(P=0.49)$ or severe RI $(P=0.74)$, however, males had a higher eGFR compared to females in the no RI group $(P=0.006)$.

Overall a higher number of patients were recruited in the intensive arm compared to the non-intensive treatment arm, this was more evident in patients presenting with no RI at diagnosis compared to those who presented with mild to moderate RI or severe RI $(P<0.001)$. Patients entering the intensive arm with no RI had a higher eGFR at diagnosis compared to those entering the non-intensive arm with no RI $(P<0.001)$. There was no significant difference in eGFR at diagnosis between the treatment arms in patients presenting with mild to moderate RI $(P=0.86)$ or severe RI $(P=0.09)$.

\section{Paraprotein and FLC isotype distribution and eGFR}

Overall IgG myeloma was the predominant paraprotein type, however, the proportion of patients with light chain only (LCO) myeloma increased with worsening eGFR category. The median eGFR was lower for patients with LCO myeloma and IgD myeloma compared to those with other paraprotein types (LCO myeloma $49.0 \mathrm{ml} /$ $\mathrm{min} / 1.73 \mathrm{~m}^{2}$ [IQR 28.0-80.0]; IgD myeloma $49.0 \mathrm{ml} /$ $\mathrm{min} / 1.73 \mathrm{~m}^{2}$ [IQR 36.7-83.0]; IgG myeloma $62.0 \mathrm{ml} /$ $\mathrm{min} / 1.73 \mathrm{~m}^{2}$ [IQR 47.0-79.0]; IgA myeloma $64.0 \mathrm{ml} /$ $\mathrm{min} / 1.73 \mathrm{~m}^{2}$ [IQR 49.0-80.0]; and IgM myeloma $65.0 \mathrm{ml} / \mathrm{min} / 1 / 73 \mathrm{~m}^{2}$ [IQR 60.5-84.5]) $(P<0.001)$. There

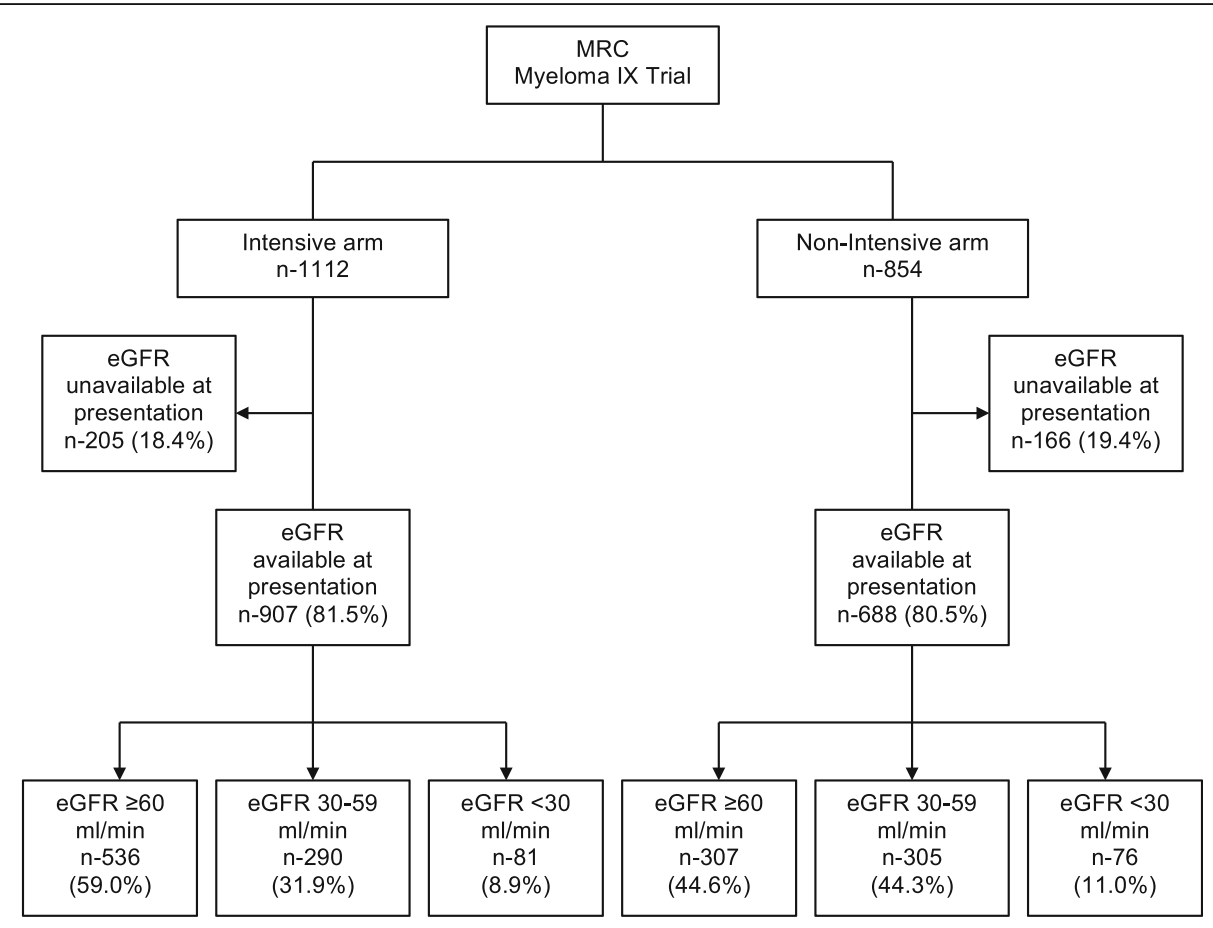

Fig. 1 CONSORT diagram showing patient distribution by renal function. (MRC, Medical Research Council) 
Table 1 Patient demographics at diagnosis

\begin{tabular}{|c|c|c|c|c|c|}
\hline Variables & $\begin{array}{l}\text { All patients } \\
(n-1595)\end{array}$ & $\begin{array}{l}\text { Patients with eGFR } \\
\geq 60 \mathrm{ml} / \mathrm{min} \\
(n-843)\end{array}$ & $\begin{array}{l}\text { Patients with eGFR } \\
30-59 \mathrm{ml} / \mathrm{min} \\
(n-595)\end{array}$ & $\begin{array}{l}\text { Patients with eGFR } \\
<30 \mathrm{ml} / \mathrm{min} \\
(n-157)\end{array}$ & $P$ value \\
\hline Median eGFR at diagnosis (ml/min/1.73 m²) (IQR) & $61(45-79)$ & $78(68-92)$ & $48(40-54)$ & $21(16-25)$ & $<0.001$ \\
\hline \multicolumn{6}{|l|}{ Treatment arm } \\
\hline Intensive (\%) & $907(56.9)$ & $536(63.6)$ & $290(48.7)$ & $81(51.6)$ & \multirow[t]{2}{*}{$<0.001$} \\
\hline Non-intensive (\%) & $688(43.1)$ & $307(36.4)$ & $305(51.3)$ & $76(48.4)$ & \\
\hline Median age - years (IQR) & $66(58-73)$ & $64(57-70)$ & $67(60-75)$ & $67(60-73)$ & $<0.001$ \\
\hline \multicolumn{6}{|l|}{ Gender } \\
\hline Male (\%) & $959(60.1)$ & $531(62.9)$ & $342(57.4)$ & $86(54.7)$ & \multirow[t]{3}{*}{0.04} \\
\hline Female (\%) & $631(39.5)$ & $311(36.9)$ & $250(42.0)$ & $70(44.6)$ & \\
\hline Unknown (\%) & $5(0.3)$ & $1(0.1)$ & $3(0.5)$ & $1(0.6)$ & \\
\hline \multicolumn{6}{|l|}{ Myeloma Type } \\
\hline $\lg G(\%)$ & $977(61.2)$ & $534(63.3)$ & $371(62.3)$ & $72(45.8)$ & \multirow[t]{6}{*}{$<0.001$} \\
\hline $\lg A(\%)$ & $347(21.7)$ & $194(23.0)$ & $130(21.8)$ & $23(14.6)$ & \\
\hline $\lg M(\%)$ & $9(0.5)$ & $8(0.9)$ & - & $1(0.6)$ & \\
\hline $\operatorname{lgD}(\%)$ & $30(1.9)$ & $13(1.5)$ & $12(2.0)$ & $5(3.2)$ & \\
\hline LCO (\%) & $215(13.5)$ & $83(9.8)$ & $76(12.8)$ & $56(35.7)$ & \\
\hline Non-secretory myeloma (\%) & $17(1.1)$ & $11(1.3)$ & $6(1.0)$ & - & \\
\hline \multicolumn{6}{|l|}{ FLC type } \\
\hline Kappa (\%) & $1029(65.2)$ & $568(68.3)$ & $373(63.3)$ & $88(56.1)$ & \multirow[t]{2}{*}{0.006} \\
\hline Lambda (\%) & $549(34.8)$ & $264(31.7)$ & $216(36.7)$ & $69(43.9)$ & \\
\hline \multicolumn{6}{|l|}{ Involved FLC level (mg/L) } \\
\hline Median Kappa FLC (IQR) & $\begin{array}{l}391.20 \\
(79.51-1106.00)\end{array}$ & $\begin{array}{l}224.10 \\
(36.96-632.20)\end{array}$ & $\begin{array}{l}474.60 \\
(115.20-1427.00)\end{array}$ & $\begin{array}{l}2217.00 \\
(668.30-5424.00)\end{array}$ & \multirow[t]{2}{*}{$<0.001$} \\
\hline Median Lambda FLC (IQR) & $\begin{array}{l}503.00 \\
(90.65-1848.00)\end{array}$ & $\begin{array}{l}266.90 \\
(57.11-942.10)\end{array}$ & $\begin{array}{l}722.50 \\
(151.40-2022.00)\end{array}$ & $\begin{array}{l}3071.00 \\
(1181.00-9079.00)\end{array}$ & \\
\hline
\end{tabular}

was no significant difference in the median age of patients between the paraprotein types $(P=0.220)$. An involved kappa FLC isotype was more common than an involved lambda FLC. The median eGFR in patients with an involved lambda FLC isotype $\left(58 \mathrm{ml} / \mathrm{min} / 1.73 \mathrm{~m}^{2}\right.$ [IQR 42-77]) was lower at diagnosis compared to patients with an involved kappa FLC $\left(63 \mathrm{ml} / \mathrm{min} / 1.73 \mathrm{~m}^{2}\right.$ [IQR 47-80]) $(P=0.008)$.

\section{Serum FLC level and eGFR}

There was a sequential rise in the median involved serum FLC level by eGFR category, irrespective of the FLC isotype. There was no significant difference in the median involved FLC level at diagnosis between the FLC isotypes for any of the three eGFR categories (Fig. 2). The median involved serum FLC level for patients with LCO myeloma and IgD myeloma was significantly higher compared to patients with other paraprotein types $(P<0.001)$ (Fig. 3$)$, consistent with the lower eGFR seen in these patients.

In order to study the relationship of the varying levels of FLC in serum with renal function, we grouped

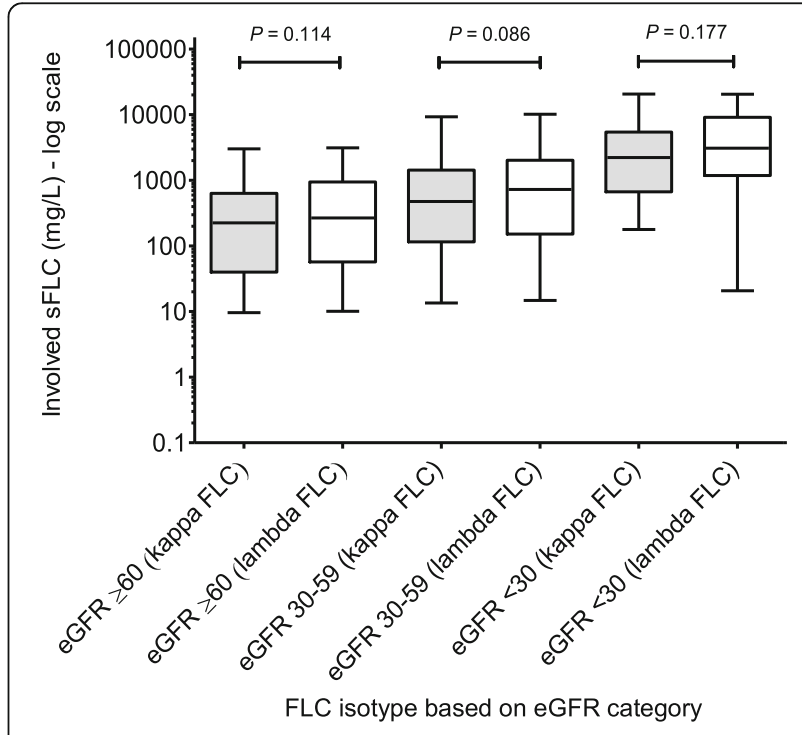

Fig. 2 Serum FLC level distribution by eGFR. (showing a progressive rise in serum FLC level with worsening of eGFR category irrespective of the FLC isotype. Data presented as box plot with whiskers and solid line represents median) 


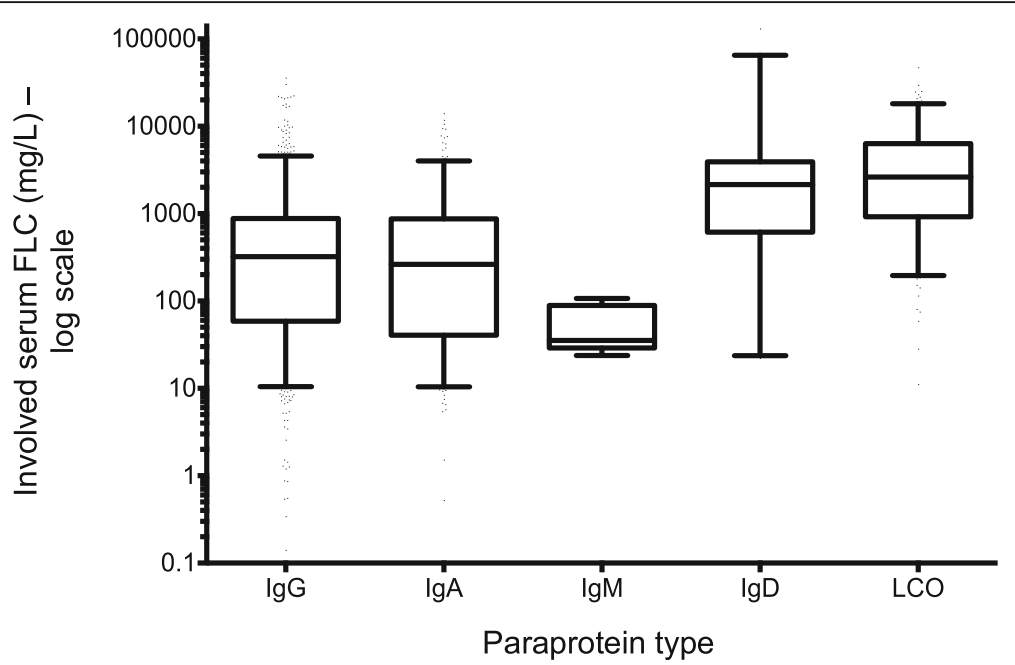

Fig. 3 Involved serum FLC level by paraprotein type. (data presented as box plot with whiskers where solid line represents median)

patients on the basis of their eGFR at diagnosis into different serum FLC level categories (Fig. 4). On comparing patients with a serum FLC level $<100 \mathrm{mg} / \mathrm{L}$ with those with a serum FLC level of 100-299 mg/L we found no difference in the proportions that had mild to moderate RI or severe RI $(P=0.82)$. For patients with a serum FLC level of 300-499 mg/L the proportion of patients with mild to moderate RI increased by approximately $6 \%$ and for severe RI by approximately $4 \%$. There was no further significant increase in the distribution of RI in patients with a serum FLC level of $500-799 \mathrm{mg} / \mathrm{L}(P=0.94)$. However, as the serum FLC level increased $>800 \mathrm{mg} / \mathrm{L}$ there was an exponential increase in the percentage of patients presenting with severe RI in comparison to those with a serum FLC level $<800 \mathrm{mg} / \mathrm{L}(P<0.001)$.

We tested the association between serum FLC level at diagnosis and severe RI by calculating the composite score for sensitivity and specificity as determined by the ROC curve analysis. The AUC was 0.80 (95\% CI $0.77-0.84 ; P<0.001)$, indicating good predictive value for an eGFR $<30 \mathrm{ml} / \mathrm{min} / 1.73 \mathrm{~m}^{2}$ (Fig. 5). A serum FLC level of $500 \mathrm{mg} / \mathrm{L}$, the level recommended by consensus for the consideration of AKI due to MCN, was associated with a sensitivity of $84 \%$ and specificity of $60 \%$. However, the optimal FLC cut-off for prediction of eGFR $<30 \mathrm{ml} / \mathrm{min} / 1.73 \mathrm{~m}^{2}$ was

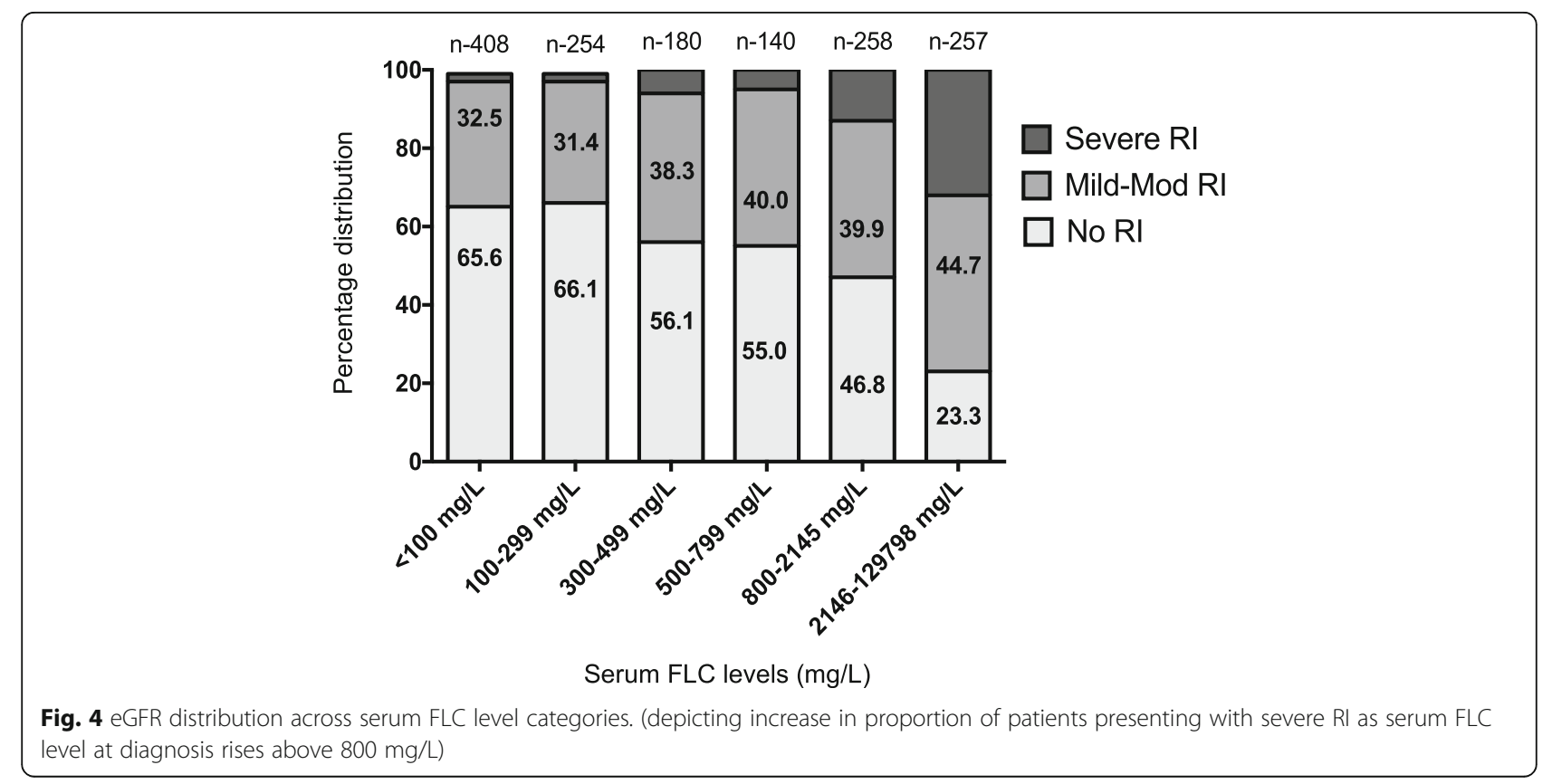




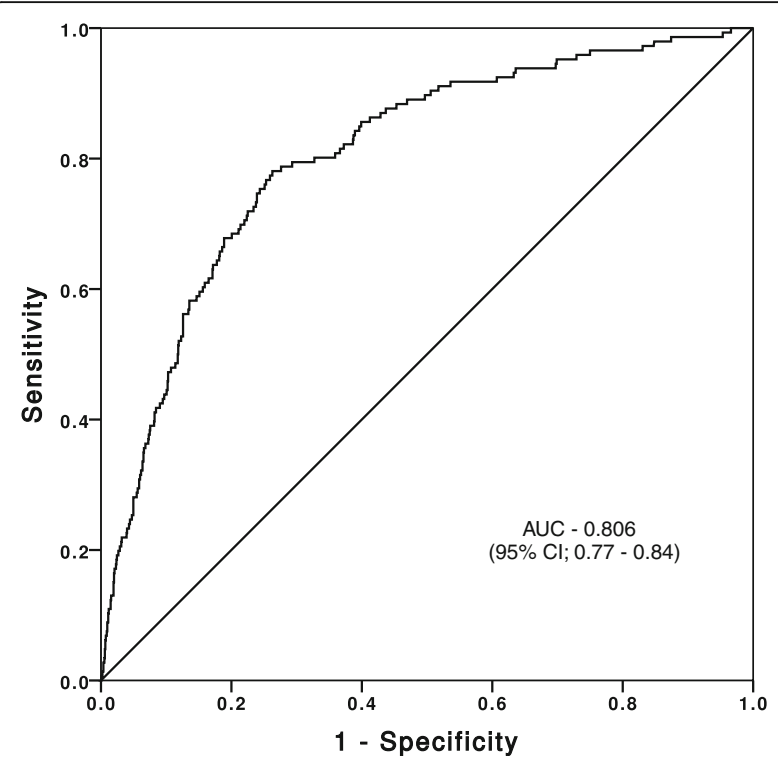

Fig. 5 Receiver operating characteristic (ROC) curve testing association between FLC level and eGFR $<30 \mathrm{ml} / \mathrm{min} / 1.73 \mathrm{~m}^{2}$. (AUC, area under curve; $\mathrm{Cl}$, confidence interval)

$800 \mathrm{mg} / \mathrm{L}$ which had a sensitivity of $80 \%$ and a specificity of $70 \%$.

\section{Correlations and regression modeling}

There were significant correlations between age $\left(\mathrm{r}_{\mathrm{s}}-\right.$ 0.174; $P<0.001$ ) and $\log _{10}$ transformed serum FLC level $\left(\mathrm{r}_{\mathrm{s}}-0.328 ; P<0.001\right)$ with eGFR at diagnosis. Multivariable linear regression modeling incorporating all factors that were associated with eGFR at diagnosis showed a significant independent association with $\log _{10}$ transformed serum FLC level, age, and female gender but not for the paraprotein type or the FLC isotype (Table 2).

Table 2 Factors associated with eGFR at diagnosis

\begin{tabular}{lllll}
\hline Factors & \multicolumn{2}{c}{ Multivariable linear regression } \\
\cline { 2 - 4 } & \multicolumn{3}{c}{$95 \%$ confidence intervals } & \\
\cline { 2 - 4 } & Beta & Lower & Upper & P value \\
\hline Age & -0.277 & -0.478 & -0.077 & 0.007 \\
Log $_{10}$ serum FLC level & -13.305 & -15.449 & -11.162 & $<0.001$ \\
Female gender & -3.271 & 0.523 & 6.019 & 0.02 \\
IgA Myeloma & 0.406 & -2.881 & 3.693 & 0.80 \\
IgM Myeloma & 7.802 & -13.357 & 28.961 & 0.47 \\
IgD Myeloma & -0.359 & -10.203 & 9.486 & 0.94 \\
LCO Myeloma & 1.855 & -1.663 & 5.373 & 0.30 \\
Kappa FLC isotype & 1.198 & -1.634 & 4.029 & 0.40 \\
Intensive arm & 3.136 & -1.023 & 7.295 & 0.13 \\
\hline
\end{tabular}

(Male gender, lgG myeloma, and non-intensive arm are reference variables)

\section{Discussion}

The purpose of this study was to investigate the relationship between renal function, demographic and myeloma characteristics, with a focus on serum FLC levels, in newly diagnosed patients with MM. Using data collected for the MRC Myeloma IX trial, we confirm a direct relationship between the serum FLC level and the severity of renal impairment. The risk of severe RI as defined by an eGFR $<30 \mathrm{ml} / \mathrm{min} / 1.73 \mathrm{~m}^{2}$ at diagnosis, only became substantial when the involved serum FLC level was > $800 \mathrm{mg} / \mathrm{L}$.

Renal impairment at diagnosis is an important, potentially modifiable risk factor in patients with MM [16]. In the MRC Myeloma IX trial, patients requiring dialysis or at a high-risk of requiring dialysis were excluded from recruitment. Despite this $47 \%$ of patients recruited in the study had an eGFR $<60 \mathrm{ml} / \mathrm{min} / 1.73 \mathrm{~m}^{2}$.

There are no kidney biopsy series that have systematically reported findings from newly diagnosed MM patients with mild to moderate RI. Thus, in most patients with MM it is not known to what proportion the mild to moderate RI at diagnosis is attributable to a) nephrotoxicity of FLC secreted by the myeloma clone, b) other myeloma-related causes of RI, and/or c) longstanding and largely irreversible RI due to unrelated comorbidities. In this study, the proportion of patients with mild to moderate RI at diagnosis was $37.3 \%$, which compares to a CKD prevalence of $25 \%$ for age- and gender-matched populations from which these MM patients were recruited $[9,17]$. This is consistent with two-thirds of the mild to moderate RI in this cohort being of longstanding origin and unrelated to the new diagnosis of MM. On assessing patients with mild to moderate RI for serum FLC level at diagnosis we found mild to moderate RI in $32.5 \%$ of patients with a serum FLC level $<100 \mathrm{mg} / \mathrm{L}$, $31.4 \%$ with a serum FLC level 100-299 mg/L and 38.3\% with a serum FLC level 300-499 mg/L. Only at a serum FLC level $>2146 \mathrm{mg} / \mathrm{L}$ was there a significant increment in the proportion of patients $(44.7 \%)$ with mild to moderate RI $(P<0.001)$ (Fig. 4). We interpret these findings as indicating that only around $12 \%$ of MM patients with mild to moderate RI may have had RI secondary to FLC nephrotoxicity and in the remaining patients, RI was most likely due to other causes.

In patients with MM and severe RI at diagnosis, renal biopsy studies have shown that the predominant lesion in up to $90 \%$ of patients is MCN, which is a direct consequence of excess monoclonal FLC production [18]. The percentage of patients presenting with severe RI in the current study was approximately five-fold the reported prevalence of severe RI in an age- and gender-matched adult population $[8,9]$. This is consistent with most of the severe RI observed in this study being attributable to $\mathrm{MM}$ rather than any pre-existing unrelated comorbidities. Also, all patients presenting 
with RI at MM diagnosis were investigated and treated for other myeloma-related causes of RI, such as dehydration and hypercalcaemia, prior to their recruitment in the MRC Myeloma IX trial.

FLCs have differential nephrotoxicity. In studies that have reported on MCN, the FLC level in serum associated with the lesion can range by 100-fold; conversely, some patients have no RI despite very high levels of FLC. The current study reinforces this observation; 19 (2.2\%) of the 843 patients with no RI had levels of serum FLC above $5000 \mathrm{mg} / \mathrm{L}$. Animal models and some previous clinical reports also indicate that $\mathrm{MCN}$ can develop in patients at far lower serum FLC levels function [19], indicating that nephrotoxicity of FLC varies greatly between patients.

LCO myeloma in the current study was associated with a lower eGFR compared to other paraprotein isotypes; this was specifically related to the serum FLC level and is not a function of the paraprotein isotype. This finding is consistent with a previous study, which reported higher urinary FLC levels in patients with LCO myeloma compared to patients with IgG myeloma and IgA myeloma [20]; in that study, RI was directly related to the urinary FLC levels. IgD myeloma was found in $1.8 \%$ of patients in the current study and the association of RI with elevated serum FLC levels at diagnosis was present in this group of patients as well. This and the possibility that IgD myeloma may still be diagnosed later than other myeloma types is under investigation in a larger cohort of patients.

$78.4 \%$ of patients with LCO myeloma had a serum FLC level $>800 \mathrm{mg} / \mathrm{L}$ and $68.2 \%$ of these had RI. In comparison, only $27.2 \%$ of patients with an intact paraprotein MM had a serum FLC $>800 \mathrm{mg} / \mathrm{L}$ and $63.2 \%$ of these patients had RI, further illustrating that the relationship with renal function is related to the FLC level in serum and not the paraprotein type. This finding was also confirmed in a multivariable linear regression model where serum FLC level was the factor with the strongest association with eGFR; this relationship was independent of age and gender at diagnosis.

This study has a few limitations: in 371 patients no samples for renal function tests were sent for central laboratory analysis and therefore these patients had to be excluded from this study. Also, patients receiving or at high risk of requiring dialysis were not recruited, as a result, the association between FLC induced nephrotoxicity and serum FLC level could not be evaluated in dialysis patients. Renal biopsy data were not available and hence we were unable to correlate serum FLC levels with myeloma cast nephropathy and other renal pathologies. Lastly, these results are only applicable to the Freelite ${ }^{\bullet}$ assay and have not been validated in the other available FLC assays [21, 22].

\section{Conclusions}

In conclusion, we report a strong relationship between higher serum FLC levels at diagnosis and the severity of RI, with a greater sensitivity and specificity for an FLC level $>800 \mathrm{mg} / \mathrm{L}$ with severe RI, irrespective of the paraprotein type. Further studies are now required to more accurately evaluate the impact of the early reduction of high serum FLC levels on RI in patients with MM, and to assess if this is associated with better long-term renal function and overall survival.

\section{Abbreviations \\ AKI: Acute Kidney Injury; AUC: Area under curve; Cl: Confidence interval; CKD: Chronic kidney disease; FLC: Free light chain; Ig: Immunoglobulin; IQR: Interquartile range; LCO: Light chain only; MCN: Myeloma cast nephropathy; MM: Multiple myeloma; MRC: Medical research council; RI: Renal impairment; ROC: Receiver operating curve; $\mathrm{r}^{\text {s: }}$ Spearmans coefficient; SD: Standard Deviation}

\section{Funding}

Financial support for the Myeloma IX trial was obtained from the Medical Research Council (G0100132).

\section{Availability of data and materials}

The data used and analysed during the current study was provided by the authors of the trial and are available from the corresponding author on reasonable request.

\section{Authors' contribution}

PY, MTD, PC, and MC conceived and designed the study; PY, MTD, PC, MC, $J P, H G, Y S A, D C, R G O, F E D, G H J, J A C$, and GJM collected and assembled the data; PY, MTD, PC, MC, JP, and DC analysed and interpreted the data; PY, MTD, and PC prepared the first draft of the manuscript; and all authors contributed to writing of the manuscript and gave final approval of the manuscript.

\section{Ethics approval and consent to participate}

MRC Myeloma IX trial had a multicentre research ethics committee and local ethics committee's approval and all patients gave written informed consent in accordance to the Declaration of Helsinki (International Standard Randomised Controlled Trial Number 68454111).

\section{Consent for publication}

Not applicable.

\section{Competing interests}

University of Birmingham and MTD own shares in Abingdon Health that manufactures immunodiagnostic tests to quantify FLC levels. PC is a medical advisor to The Binding Site that produces the Freelite assay. The remaining authors have no competing financial interests.

\section{Publisher's Note}

Springer Nature remains neutral with regard to jurisdictional claims in published maps and institutional affiliations.

\footnotetext{
Author details

${ }^{1}$ Department of Renal Medicine, University Hospital Birmingham NHS Foundation Trust, Birmingham, UK. ${ }^{2}$ Institute of Immunology and Immunotherapy, University of Birmingham, Birmingham, UK. ${ }^{3}$ Department of Haematology, University Hospital Birmingham NHS Foundation Trust, Birmingham, UK. ${ }^{4}$ Clinical Trials Research Unit, University of Leeds, Leeds, UK. ${ }^{5}$ Department of Haematology, Leeds Teaching Hospitals NHS Trust, Leeds, UK. ${ }^{6}$ Myeloma Research Centre, Division of Molecular Pathology, The Institute of Cancer Research, London, UK. ${ }^{7}$ Myeloma Institute of Research and Therapy, University of Arkansas for Medical Sciences, Little Rock, AR, USA. ${ }^{8}$ Department of Haematology, University of Newcastle, Newcastle-upon-Tyne, UK. ${ }^{9}$ Clinical Immunology Service, College of Medical and Dental Sciences, University of Birmingham, Edgbaston, Birmingham B15 2TT, UK.
} 
Received: 23 January 2018 Accepted: 25 June 2018

Published online: 13 July 2018

\section{References}

1. Knudsen LM, Hjorth M, Hippe E. Renal failure in multiple myeloma: reversibility and impact on the prognosis. Nordic Myeloma Study Group European journal of haematology. 2000;65(3):175-81.

2. Dimopoulos MA, Kastritis E, Rosinol L, Blade J, Ludwig H. Pathogenesis and treatment of renal failure in multiple myeloma. Leukemia : official journal of the Leukemia Society of America, Leukemia Research Fund, UK. 2008;22(8): 1485-93.

3. Kleber M, Ihorst G, Terhorst M, Koch B, Deschler B, Wasch R, Engelhardt M. Comorbidity as a prognostic variable in multiple myeloma: comparative evaluation of common comorbidity scores and use of a novel MMcomorbidity score. Blood cancer journal. 2011;1(9):e35.

4. Song X, Cong Z, Wilson K. Real-world treatment patterns, comorbidities, and disease-related complications in patients with multiple myeloma in the United States. Curr Med Res Opin. 2016;32(1):95-103.

5. Stringer $S$, Cook M, Cockwell P. Achieving an early myeloma response in patients with kidney impairment. Adv Chronic Kidney Dis. 2012;19(5):303-11.

6. Roussou M, Kastritis E, Migkou M, Psimenou E, Grapsa I, Matsouka C, Barmparousi D, Terpos E, Dimopoulos MA. Treatment of patients with multiple myeloma complicated by renal failure with bortezomib-based regimens. Leuk Lymphoma. 2008;49(5):890-5.

7. Hutchison CA, Cockwell P, Stringer S, Bradwell A, Cook M, Gertz MA Dispenzieri A, Winters JL, Kumar S, Rajkumar SV, et al. Early reduction of serum-free light chains associates with renal recovery in myeloma kidney. J Am Soc Nephrol. 2011;22(6):1129-36.

8. Jain P, Calvert M, Cockwell P, McManus RJ. The need for improved identification and accurate classification of stages 3-5 chronic kidney disease in primary care: retrospective cohort study. PLoS One. 2014;9(8): e100831.

9. Zoccali C, Kramer A, Jager KJ. Epidemiology of CKD in Europe: an uncertain scenario. Nephrology, dialysis, transplantation : official publication of the European Dialysis and Transplant Association - European Renal Association. 2010;25(6):1731-3.

10. Dimopoulos MA, Delimpasi S, Katodritou E, Vassou A, Kyrtsonis MC, Repousis P, Kartasis Z, Parcharidou A, Michael M, Michalis E, et al. Significant improvement in the survival of patients with multiple myeloma presenting with severe renal impairment after the introduction of novel agents. Annals of oncology : official journal of the European Society for Medical Oncology / ESMO. 2014:25(1):195-200.

11. Torra R, Blade J, Cases A, Lopez-Pedret J, Montserrat E, Rozman C, Revert L. Patients with multiple myeloma requiring long-term dialysis: presenting features, response to therapy, and outcome in a series of 20 cases. $\mathrm{Br}$ J Haematol. 1995:91(4):854-9.

12. Morgan GJ, Gregory WM, Davies FE, Bell SE, Szubert AJ, Brown JM, Coy NN, Cook G, Russell NH, Rudin C, et al. The role of maintenance thalidomide therapy in multiple myeloma: MRC myeloma IX results and meta-analysis. Blood. 2012;119(1):7-15.

13. Morgan GJ, Davies FE, Gregory WM, Bell SE, Szubert AJ, Navarro Coy N, Cook G, Feyler S, Johnson PR, Rudin C, et al. Cyclophosphamide, thalidomide, and dexamethasone as induction therapy for newly diagnosed multiple myeloma patients destined for autologous stem-cell transplantation: MRC myeloma IX randomized trial results. Haematologica. 2012;97(3):442-50

14. Morgan GJ, Davies FE, Gregory WM, Russell NH, Bell SE, Szubert AJ, Navarro Coy N, Cook G, Feyler S, Byrne JL, et al. Cyclophosphamide, thalidomide and dexamethasone (CTD) as initial therapy for patients with multiple myeloma unsuitable for autologous transplantation. Blood. 2011;118(5): 1231-8.

15. Morgan GJ, Davies FE, Gregory WM, Cocks K, Bell SE, Szubert AJ, NavarroCoy N, Drayson MT, Owen RG, Feyler S, et al. First-line treatment with zoledronic acid as compared with clodronic acid in multiple myeloma (MRC myeloma IX): a randomised controlled trial. Lancet. 2010;376(9757):1989-99.

16. MacLennan IC, Falconer-Smith JF, Crockson RA, Cooper EH, Knight FR, Cuzick J, Hardwicke J. Analysis and management of renal failure in fourth MRC myelomatosis trial. MRC working party on leukaemia in adults. $\mathrm{Br}$ Med J (Clin Res Ed). 1984;288(6428):1411-6.
17. Hoerger TJ, Simpson SA, Yarnoff BO, Pavkov ME, Rios Burrows N, Saydah SH, Williams DE, Zhuo X. The future burden of CKD in the United States: simulation model for the CDC CKD initiative. American journal of kidney diseases : the official journal of the National Kidney Foundation. 2015;65(3): 403-11.

18. Hutchison CA, Heyne N, Airia P, Schindler R, Zickler D, Cook M, Cockwell P, Grima D. Immunoglobulin free light chain levels and recovery from myeloma kidney on treatment with chemotherapy and high cut-off haemodialysis. Nephrology, dialysis, transplantation : official publication of the European Dialysis and Transplant Association - European Renal Association. 2012;

19. Sanders PW, Booker BB. Pathobiology of cast nephropathy from human Bence Jones proteins. J Clin Invest. 1992;89(2):630-9.

20. Drayson M, Begum G, Basu S, Makkuni S, Dunn J, Barth N, Child JA. Effects of paraprotein heavy and light chain types and free light chain load on survival in myeloma: an analysis of patients receiving conventional-dose chemotherapy in Medical Research Council UK multiple myeloma trials. Blood. 2006;108(6):2013-9.

21. te Velthuis H, Knop I, Stam P, van den Broek M, Bos HK, Hol S, Teunissen E, Fischedick KS, Althaus H, Schmidt B, et al. N latex FLC - new monoclonal high-performance assays for the determination of free light chain kappa and lambda. Clin Chem Lab Med. 2011:49(8):1323-32.

22. Campbell JP, Cobbold M, Wang Y, Goodall M, Bonney SL, Chamba A, Birtwistle J, Plant T, Afzal Z, Jefferis R, et al. Development of a highlysensitive multi-plex assay using monoclonal antibodies for the simultaneous measurement of kappa and lambda immunoglobulin free light chains in serum and urine. J Immunol Methods. 2013;391(1-2):1-13.

\section{Ready to submit your research? Choose BMC and benefit from:}

- fast, convenient online submission

- thorough peer review by experienced researchers in your field

- rapid publication on acceptance

- support for research data, including large and complex data types

- gold Open Access which fosters wider collaboration and increased citations

- maximum visibility for your research: over $100 \mathrm{M}$ website views per year

At BMC, research is always in progress.

Learn more biomedcentral.com/submissions 